\title{
Development of a Strain Evaluation Technique Using a Micro Focus X-ray Beam
}

\author{
Takuji Kita ${ }^{1}$, Yasuo Nagano ${ }^{1}$, Hiroshi Kawamoto ${ }^{1}$ and Ryouichi Yokoyama ${ }^{2}$ \\ ${ }^{1}$ Synergy Ceramics Laboratory, Fine Ceramics Research Association, Nagoya 456-8587, Japan \\ ${ }^{2}$ X-ray Research Laboratory, Rigaku Co., Tokyo 196-8666, Japan
}

A strain evaluation technique using a micro focus X-ray beam was developed. An electron gun with two electron lenses was used in order to steadily make a fine focus of an electron beam on a copper target. A target current was $24 \mu \mathrm{A}$, and an X-ray brightness $162 \times 10^{9} \mathrm{~W} / \mathrm{m}^{2} \mathrm{was}$ obtained. X-ray beam was condensed to a converging angle $0.09 \mathrm{deg}$ with a convergent unit, and the minimum focus diameter was $60 \mu \mathrm{m}$. A $\mathrm{Cu}-$ $\mathrm{K} \alpha_{2}$ 's peak can be easily split from a K $\alpha_{1}$ 's peak, because the converging angle of the X-ray beam is low and an X-ray brightness is high. A charge coupled device camera can be set in order to observe the irradiated area on a sample. A cubic $\mathrm{ZrO}_{2}-10 \mathrm{~mol}_{2} \mathrm{Y}_{2} \mathrm{O}_{3}$ single crystal cut along a (001) plane was selected as a sample to ensure a measurement technique. The difference in a Miller index from an integer was less than $0.4 \%$. A lattice parameter of the specimen was $0.51442 \pm 0.00002 \mathrm{~nm}$, and the reference value was $0.5144 \mathrm{~nm}$. Strain was loaded to the sample with a four-point bending jig. A strain tensor was analyzed from the difference in the lattice parameters which were calculated using from 15 to 50 planes. Although the some measured values had a more than $100 \times 10^{-6}$ difference from the estimated ones, the other measured values were almost same as the estimated ones within experimental error.

(Received May 14, 2003; Accepted May 26, 2003)

Keywords: strain evaluation, residual stress, X-ray diffraction, synchrotron radiation, micro focus X-ray, cubic zirconia, single crystal

\section{Introduction}

High performance composite materials are developed for various regions, such as electrics, optics, structural and heat resistant material in order to achieve the synergistic properties. Stress is concentrated between different elastic coefficient interfaces or different thermal expansion interfaces, and the evaluation technique is required. However, the smaller and more complex the devices are, the more difficult to evaluate the stress concentration.

The $\sin ^{2} \psi$ method using X-ray, which is the most wellknown evaluation of the stress, is inadequate for the orientated structure, because the random crystal direction is assumed in this method. Hanabusa et al. ${ }^{1)}$ and Tanaka et al., ${ }^{2,3)}$ applied an improved $\sin ^{2} \psi$ diagram for the fibrous texture that is completely orientated in a thin film, but there are few studies on an incomplete orientated structure. A specimen which has large strain gradient in an X-ray irradiated region is also inadequate, because the averaged strain of a plenty of grains is detected with the $\sin ^{2} \psi$ method. From these restrictions, the irradiated diameter is required more than $300 \mu \mathrm{m}$.

A new strain-evaluation technique which overcomes these problems should be developed for the micro-structures. Suyama $e \mathrm{al}{ }^{4)}$ irradiated a micro focus X-ray beam through $\phi 1 \mathrm{~mm}$ pinhole on one grain (mean grain size $=$ approximately $20 \mathrm{~mm}$ ) of a coarsened $\mathrm{Fe}+3 \% \mathrm{Si}$ specimen, and detected the reflected X-ray with a Position Sensitive Proportional Counter. The structure in the irradiated area is a single crystal, and the principal strain distribution is calculated by the changes in the lattice spacing. However the precise strain-evaluation technology for finer crystals should be developed.

Although a high intensity and micro focus X-ray beam should be used in order to evaluate stress in a micro region, it can not be obtained except in synchrotron radiation facilities. ${ }^{5,6)}$ In this study, the strain evaluation technology using a small-scale micro focus X-ray diffraction system is developed.

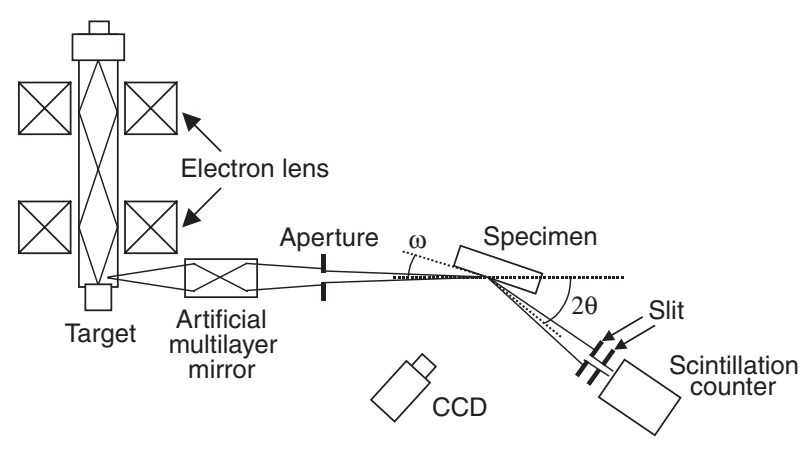

Fig. 1 Schematic diagram of the developed apparatus.

\section{Specifications of the Developed Apparatus}

Following specifications should be satisfied in order to detect reflection X-rays from one single crystal and precisely evaluate the strain.

(1) An X-ray source producing a high brightness X-ray in steady.

(2) A convergent unit producing a low converging angle and micro focus X-ray beam.

(3) A goniometer detecting diffracted X-rays in precise. An apparatus that satisfies all these specifications were developed with RIGAKU Co. A schematic diagram of the developed apparatus is shown in Fig. 1, and the specifications are mentioned below.

\subsection{X-ray source}

A rotating anode is a well-known apparatus as a high power X-ray source. For example, in the case of ultraX18 (RIGAKU Co.), the tube voltage and the target current is $40 \mathrm{kV}$ and $30 \mathrm{~mA}$, respectively. However, the X-ray brightness, which is calculated by dividing the maximum allowable load by the focus size on a target, is low, $12 \times 10^{9} \mathrm{~W} / \mathrm{m}^{2}$.

An electron gun with two electron lenses was used in order to steadily make a fine focus of the electron beam on a copper target. A single $\mathrm{LaB}_{6}$ crystal, of which the emission area is 
$\phi 200 \mu \mathrm{m}$, was used as an emission source of the electron beam, because its work function is lower and a lifetime is longer compared with tungsten. An X-ray is generated from $2.2 \times 3.36 \mu \mathrm{m}^{2}$ on the target. The target current was $24 \mu \mathrm{A}$, and the X-ray brightness $162 \times 10^{9} \mathrm{~W} / \mathrm{m}^{2}$ was obtained, when the tube voltage was $50 \mathrm{kV}$, and the tube current was $0.7 \mathrm{~mA}$.

\subsection{Convergent unit}

The X-ray generated on the target is condensed to the converging angle $0.2 \mathrm{deg}$ with an artificial multilayer mirror unit. This mirror unit consists of two mirrors made of tungsten and silicon, and condenses the X-ray by reflecting once on an each mirror.

The converging angle of the X-ray beam was decreased to $0.09 \mathrm{deg}$ with an aperture $(\phi 0.2 \mathrm{~mm})$, and the minimum focus diameter was $60 \mu \mathrm{m}$. The converging angle was measured by a profile shown in Fig. 2, of the direct beam around the $2 \theta$ origin. The focus diameters in a horizontal plane and a vertical plane were measured by scanning a $\phi 20 \mu \mathrm{m}$ pinhole around the focus position, as shown in Fig. 3. Figure 4 shows the diffraction profile of a (004) plane of a cubic $\mathrm{ZrO}_{2}-$ 10 mol\% $\mathrm{Y}_{2} \mathrm{O}_{3}$ single crystal. The $\mathrm{Cu}-\mathrm{K} \alpha_{2}$ 's peak is completely split from the $\mathrm{K} \alpha_{1}$ 's peak, because the converging angle of the X-ray beam is low and the X-ray brightness is high.

\subsection{Goniometer}

The incident angle $\omega$, and the diffraction angle $2 \theta$, are detected by rotary encoder. Their angle resolution is $10^{-4} \mathrm{deg} / \mathrm{step}$. A charge coupled device camera, which magnifies 500 times on a monitor, can be set in order to observe the irradiated area on a sample. A four-point bending jig can be set on a sample stage in order to measure the diffraction angle of the sample with a load.

\section{Experimental Procedures}

A cubic $\mathrm{ZrO}_{2}-10 \mathrm{~mol} \% \mathrm{Y}_{2} \mathrm{O}_{3}$ single crystal cut along a (001) plane $\left(5 \times 5 \times 45 \mathrm{~mm}^{3}\right)$ was selected as a sample to ensure a measurement technique. Strain $\left(300 \times 10^{-6}\right)$ was loaded to the sample with the four-point bending jig. Measuring points with X-ray and a strain gauge are shown in Fig. 5.

Strain tensor was analyzed from the difference in the lattice parameters, ${ }^{7)}$ which were calculated using from 15 to 50 planes.

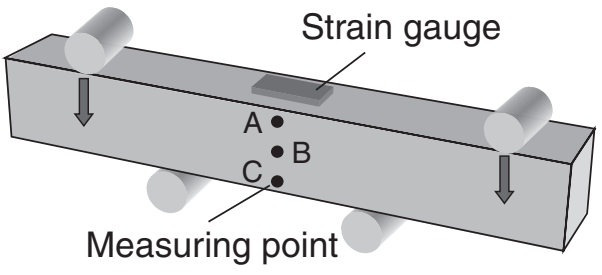

Fig. 5 Strain measuring positions on a specimen.

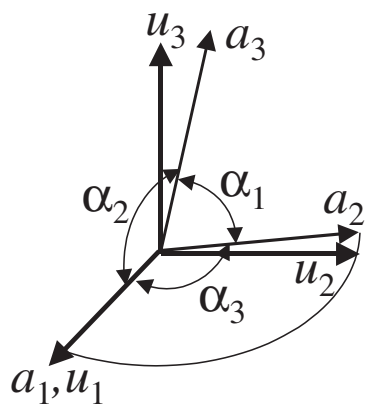

Fig. 6 Relationship between the unit cell $a_{i}$ and the Cartesian coordinates $u_{i}$.

Consider a crystal with the lattice parameters $a_{i}$ and $\alpha_{i}$ is attached to a Cartesian-coordinate system $\boldsymbol{u}_{i}$ as shown in Fig. 6 , that is the $u_{1}$ axis parallel to $a_{1}$, the $u_{2}$ axis in the plane of $a_{1}$ and $a_{2}$, and the $u_{3}$ axis perpendicular to that plane.

Real-lattice vectors in the Cartesian-coordinate system are expressed as,

$$
A=\left(\begin{array}{ccc}
a_{1} & a_{2} \cos \alpha_{3} & -a_{3} \cos \alpha_{2} \\
0 & a_{2} \sin \alpha_{3} & -a_{3} \sin \alpha_{2} \cos \beta_{1} \\
0 & 0 & 1 / b_{3}
\end{array}\right),
$$

where the $\beta_{i}$ s and $b_{i}$ s are the reciprocal-lattice parameters for the unit cell.

Let $A_{0}$ and $A_{1}$ be the matrix for the unstrained unit cell and the strained cell, respectively. A matrix $T$ which transforms from $A_{0}$ to $A_{1}$ is defined as follows,

$$
\boldsymbol{A}_{1}=\boldsymbol{T} \boldsymbol{A}_{0} .
$$

From the definition of the strain tensor $\varepsilon_{i j}$, the strain tensor in the Cartesian-coordinate system is given by

$$
\boldsymbol{\varepsilon}_{i j}=\left(\boldsymbol{T}_{i j}+\boldsymbol{T}_{j i}\right) / 2-\boldsymbol{I}_{i j},
$$

where $\boldsymbol{I}$ is a unit matrix.

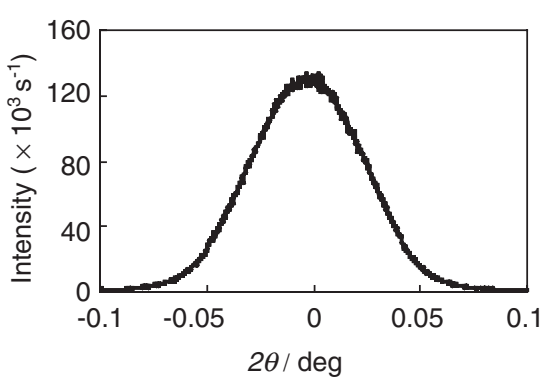

Fig. 2 Direct beam profile around $2 \theta$ origin.

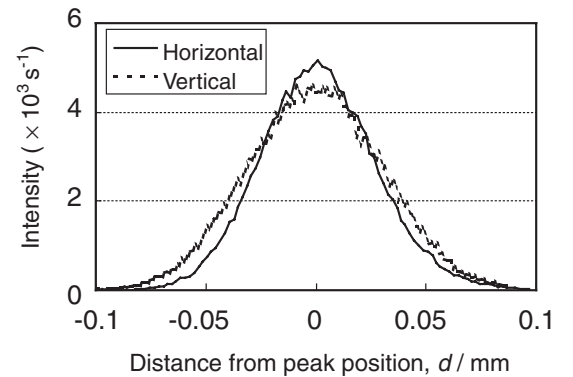

Fig. 3 Dimension of direct beam at focus position.

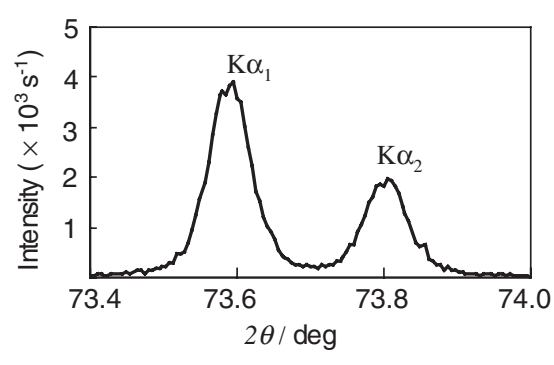

Fig. 4 Diffraction pattern of $\mathrm{ZrO}_{2}-10 \mathrm{~mol} \%$ $\mathrm{Y}_{2} \mathrm{O}_{3}$ (004) plane. 

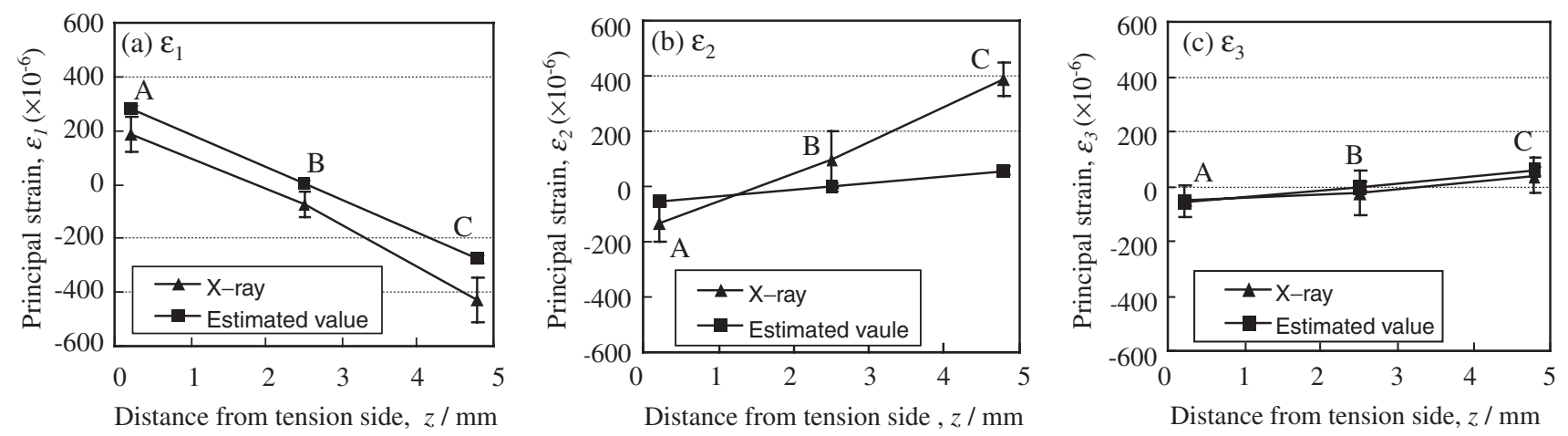

Fig. 7 Principal strain distribution.

The strain tensor contains both a hydrostatic strain $\Delta / 3$ and a distortion strain term, therefore eq. (3) can be written in terms of the two components

$$
\boldsymbol{\varepsilon}=\boldsymbol{\varepsilon}_{d}+\frac{\Delta}{3} \boldsymbol{I},
$$

where $\Delta=\varepsilon_{11}+\varepsilon_{22}+\varepsilon_{33} . \boldsymbol{\varepsilon}_{d}$ is the distortional term,

$$
\boldsymbol{\varepsilon}_{d}=\left(\begin{array}{ccc}
\varepsilon_{11}-\Delta / 3 & \varepsilon_{12} & \varepsilon_{13} \\
\varepsilon_{12} & \varepsilon_{22}-\Delta / 3 & \varepsilon_{23} \\
\varepsilon_{13} & \varepsilon_{23} & \varepsilon_{33}-\Delta / 3
\end{array}\right) .
$$

Principal strains, $\varepsilon_{1}, \varepsilon_{2}$ and $\varepsilon_{3}$, are calculated as eigenvalues of $\boldsymbol{\varepsilon}_{d}$.

\section{Results}

A part of the result of a crystal orientation analysis for the specimen without a load is shown in Table 1. All the diffraction intensity exceeds $500 \mathrm{~s}^{-1}$, and it follows that an extremely high brightness X-ray is irradiated on the specimen. The differences in the Miller indices from integers were less than $0.4 \%$. The lattice parameter of the specimen was $0.51442 \pm 0.00002 \mathrm{~nm}$, and the reference value was $0.5144 \mathrm{~nm}^{8}{ }^{8}$ From these results, it follows that a crystal orientation and lattice parameters can be extremely precisely analyzed by the developed apparatus.

Principal strains of each measuring point were calculated using from eqs. (1) to (5). A principal strain distribution with a load is shown in Fig. 7. Errors of the principal strains were evaluated from errors of the lattice parameters. A principal strain estimated from the strain gauge's value and the crystal orientation is also plotted in Fig. 7. Although the some

Table 1 Reflection index of $\mathrm{ZrO}_{2}-10 \mathrm{~mol} \% \mathrm{Y}_{2} \mathrm{O}_{3}$.

\begin{tabular}{cccc}
\hline \multicolumn{3}{c}{ Miller index } & Intenstiy, \\
\cline { 1 - 3 }$h$ & $k$ & $l$ & 3313 \\
\hline 0.000 & 0.000 & 4.000 & 1061 \\
0.998 & -1.001 & 5.000 & 836 \\
0.001 & 0.000 & 6.000 & 640 \\
4.000 & -0.001 & 4.000 & 826 \\
2.999 & -1.001 & 5.000 & 1287 \\
2.001 & -0.001 & 5.999 & 1106 \\
-0.004 & -1.999 & 6.000 & 515 \\
4.001 & 1.999 & 3.999 & \\
\hline
\end{tabular}

measured values had a more than $100 \times 10^{-6}$ difference from the estimated ones, the other measured values were almost same as the estimated ones within experimental error. In conclusion, the micro focus X-ray diffraction system can be used for strain evaluation in micro region.

\section{Summary}

Strain evaluation technology which can be used in a smallscale system was developed.

(1) An X-ray source which produced high brightness X-ray in steady was obtained. The target current was $24 \mu \mathrm{A}$, and the X-ray brightness was $162 \times 10^{9} \mathrm{~W} / \mathrm{m}^{2}$.

(2) A convergent unit which produced a low converging angle $(0.09 \mathrm{deg})$ and a fine focus X-ray beam $(\phi 60 \mu \mathrm{m})$ was installed.

(3) A goniometer which detects reflection X-rays with an angle resolution for $2 \theta$ and $\omega$ of $10^{-4} \mathrm{deg} / \mathrm{step}$ was used.

(4) The principal strain distribution in a cubic $\mathrm{ZrO}_{2}-$ $10 \mathrm{~mol} \% \mathrm{Y}_{2} \mathrm{O}_{3}$ single crystal was measured. Most of the measured values were almost same as the estimated ones within experimental error.

\section{Acknowledgments}

Research supported by NEDO, under the Synergy Ceramics Project promoted by METI, Japan. The authors thank to Mr. Oguiso for his considerable contribution in the development of the present system during his stay in X-ray Research Laboratory, Rigaku Co. as a general manager.

\section{REFERENCES}

1) T. Hanabusa, K. Tominaga and H. Fujiwara: J. Soc. Mater. Sci., Japan 42 (1993) 90-96.

2) K. Tanaka, K. Ishihara and K. Inoue: J. Soc. Mater. Sci., Japan 45 (1996) 945-950.

3) K. Tanaka, Y. Akiniwa, T. Ito and K. Inoue: JSME Int. J. 42 (1999) 224234.

4) Y. Suyama, S. Ohya and Y. Yoshioka: J. Soc. Mater. Sci., Japan 48 (1999) 1437-1442.

5) S. Takago, T. Sasaki, K. Akita, Y. Yoshioka and Y. Hirose: J. Soc. Mater. Sci., Japan 49 (2000) 729-734.

6) K. Akita, Y. Yoshioka, H. Suzuki and T. Sasaki: J. Soc. Mater. Sci., Japan 49 (2000) 722-728.

7) Jin-Seok Chung and Gene E. Ice: J. Appl. Phys. 86 (1999) 5249.

8) Y. Murakami, I. Nagano, H. Yamamoto and H. Sakata: J. Mater. Sci. Lett. 16 (1997) 1686. 